\title{
Rock Mass Blastability Classification Using Fuzzy Pattern Recognition and the Combination Weight Method
}

\author{
Shuangshuang Xiao, Kemin Li, Xiaohua Ding, and Tong Liu \\ State Key Laboratory of Coal Resources and Safe Mining, School of Mines, China University of Mining and Technology, \\ Xuzhou, Jiangsu 221116, China
}

Correspondence should be addressed to Kemin Li; likemin515@hotmail.com

Received 27 January 2015; Accepted 27 May 2015

Academic Editor: Rama S. R. Gorla

Copyright (C) 2015 Shuangshuang Xiao et al. This is an open access article distributed under the Creative Commons Attribution License, which permits unrestricted use, distribution, and reproduction in any medium, provided the original work is properly cited.

\begin{abstract}
Rock mass blastability classification provides a theoretical basis for rock mass blasting design, which is used to select blasting explosives, to estimate the unit explosive consumption, and to determine blasting design parameters. The primary factors that affect rock mass blastability were analyzed by selecting five indexes for rock mass blastability classification, that is, the rock Protodyakonov coefficient, rock tensile strength, rock density, rock wave impedance, and integrity coefficient of rock mass, and by identifying standards for the rock mass blastability classification and a method for testing the blasting classification indexes. The index weights were calculated using the combination weight method, which is based on game theory. A model for rock mass blastability classification was developed in combination with a fuzzy pattern recognition method. This classification method was applied to a Heidaigou open-pit coal mine, where mudstone, fine sandstone, medium sandstone, and coarse sandstone were determined to have a blastability degree of II, which corresponds to a blastability characterization of "easy," and the unit explosive consumption of mudstone, fine sandstone, medium sandstone, and coarse sandstone was determined to be $0.44,0.42,0.40$, and $0.36 \mathrm{~kg} / \mathrm{m}^{3}$, respectively. These results were used to develop a loose blasting design that was effective for loose blasting.
\end{abstract}

\section{Introduction}

Rock mass blastability is a measure of the resistance of a rock mass to blasting and crushing. The physical and mechanical properties and structural characteristics of rocks synchronize to various extents and in different ways to impede blasting and crushing under blasting loading [1]. Thus, the rock mass blastability is also a comprehensive indicator of several inherent properties of a rock mass under dynamic loading. The rock mass blastability reflects the degree of difficulty in rock blasting [2]. An understanding of rock mass blastability and systematic rock mass blastability classifications form the theoretical basis of blasting optimization design [3]. The rock mass blastability can be used to select suitable explosives, estimate the explosive unit consumption, and determine reasonable blasting parameters, which can reduce blasting costs and improve labor productivity by ensuring predictable blasting characteristics.

Foreign and Chinese scholars have conducted numerous research studies on rock mass blastability classification using different methods from various perspectives and have developed a variety of indexes and methods for rock mass blastability classification [4]. There are currently two methods for rock mass blastability classification. In the first method, the analysis and calculation involve one or more parameters, and a numerical value, such as a blastability index or the crushing energy, is chosen as a measure of the blastability of the rock mass [5]. In the second method, various parameters are chosen to describe the rock mass, and the rock mass blastability classification is performed using statistical mathematics, fuzzy mathematics, or other mathematical methods. The characteristics and internal mechanisms that affect rock mass blastability can be identified more accurately using several indexes to systematically evaluate the rock mass blastability. Therefore, many classification schemes and evaluation algorithms have been applied to rock mass blastability classification, including neural networks [6-8], projection pursuit [9], genetic algorithms [10], fuzzy set theory [1113], cluster analysis [14], and attribute recognition [15]. Each 
algorithm has its advantages and disadvantages. For example, a neural network has considerable fault-tolerance ability and a rapid evaluation speed but requires a representative learning sample. In addition, the learning parameters and number of hidden layers are difficult to identify, and the number of hidden layers affects the convergence rate, the convergence properties of the network, and its applicability to nonlinear problems. The index weights do not need to be identified when the rock mass blastability is classified using the projection pursuit algorithm, thereby ensuring that the classification is objective. However, when optimizing the projection direction, this scheme can easily converge to a local optimum, which results in early maturing or early convergence, among other problems. Genetic algorithms can be used to accurately classify the respective categories but has additional parameter requirements, such as gene variables and genetic generations. The challenge encountered in using cluster analysis and attribute recognition is to determine reasonable index weights.

There are three essential requirements for developing a rock mass blastability classification model. First, the most representative characteristic must be chosen as the classification index, and classification standards must be developed. Second, each index should be assigned a reasonable weight. Finally, a suitable evaluating algorithm should be chosen. The rock blasting mechanism and the factors affecting rock mass blastability for the aforementioned research scenario were used to identify the classification indexes and classification standards for rock mass blastability. The blastability of a rock mass was described using the following values: "easy," "moderate," "difficult," and other fuzzy values, depending on practical production requirements. The indices of two rock samples typically have similar values but are characterized by different rating categories by observation. This result is not reasonable. Thus, rock blastability can be characterized using transitional values that lie in between different levels; that is, the values are fuzzy. There is no distinct boundary between different levels. The same rock mass could be assigned to different classifications by different people or based on different situations. Thus, it is more suitable to use fuzzy mathematics to classify rock mass blastability. Rock mass classification can then be based on this developed rock mass rating and the rock characteristics; that is, rock mass blastability classification is a pattern recognition problem. Therefore, fuzzy pattern recognition was used to develop a rock mass blastability classification model. However, the weights of the indexes are not considered in pattern recognition, which prevents the application of this method to cases with unequal index weights. The combination weight method was used to identify the index weights to reduce the effects of subjective factors and avoid irrelevant factors.

\section{Indexes and Standards for the Classification of Rock Mass Blastability}

2.1. Selection of Classification Index. Explosive blasting can fracture a rock mass in two ways. First, the cohesive force between rock granules can be overcome, thereby rupturing the internal rock structure and producing a new fracture surface. Second, primary and secondary fractures can be exacerbated via further expansion. Therefore, the primary influential factors of rock mass blastability are the physical and mechanical properties of the rock and the structural characteristics of the rock mass [16]. Typical indexes for classifying rock mass blastability include the rock density, rock wave impedance, rock tensile strength, integrity coefficient of the rock mass, and the mean crack interval of the rock mass. These indexes reflect different aspects of rock mass blastability. However, to simplify rock mass blastability classification and enable its practical application, all of the indexes are not used. The characteristics of a rock mass must be considered when choosing indexes for rock mass blastability classification. Minor representative indexes can comprehensively reflect different aspects of the rock mass blastability. There should be little or no correlation between the indexes. The chosen indexes should be easy to obtain using various methods, such as experiments and field measurements. The aforementioned considerations were used to select the following final indexes for when considering rock mass blastability.

\subsubsection{Protodyakonov Coefficient and Tensile Strength of Rock.} The shock wave and detonation gas produced by explosive blasting can typically rupture a rock mass through pulling and pressing. Therefore, the Protodyakonov coefficient and tensile strength of the rock are important parameters in rock mass blastability. During blasting, the rock is subject to temporary impact loading, for which the rock dynamic loading strength is clearly higher than the rock static loading strength. Therefore, the rock mass blastability can be accurately measured by indexes for the dynamic loading strength that are affected by the triaxial effect of the rock. However, the dynamic loading strength of rock is difficult to measure and exhibits a strong linear correlation with the uniaxial static loading compression strength and tensile strength [17]. Thus, the static loading strength is chosen as one of the indexes for rock mass blastability classification. The Protodyakonov coefficient of the rock, which is determined from the uniaxial compressive strength of the rock (1), is an objective measure of rock fastness that is widely applied in China. Therefore, the Protodyakonov coefficient and compressive strength of the rock are chosen as indexes for rock mass blastability classification:

$$
f=\frac{\sigma_{p}}{K} .
$$

In the equation above, $f$ is the Protodyakonov coefficient of the rock; $\sigma_{p}$ is the rock's uniaxial compressive strength in $\mathrm{MPa}$; and $K$ is a constant equal to $10 \mathrm{MPa}$.

2.1.2. Rock Density. The energy produced from rock blasting is transferred into kinetic energy in the rock block, which can result in the displacement or thrusting of the rock block. A higher rock density causes more of the energy produced in rock blasting to be consumed by the displacement and thrusting of the rock. Therefore, the amount of energy consumed is indicative of the difficulty of the rock blast; that is, the rock mass blastability decreases with increasing rock 
TABLE 1: Classification standards of rock mass blastability.

\begin{tabular}{lcccccc}
\hline Blastability class & $\begin{array}{c}\sigma_{t} \\
(\mathrm{MPa})\end{array}$ & $f$ & $\begin{array}{c}P \\
\left(\mathrm{t} / \mathrm{m}^{3}\right)\end{array}$ & $K_{v}$ & $\begin{array}{c}Z \\
\left(10^{6} \mathrm{~kg} / \mathrm{m}^{3} \times \mathrm{m} / \mathrm{s}\right)\end{array}$ & Characterization of blastability \\
\hline I & $\leq 1.5$ & $\leq 2.5$ & $\leq 2.0$ & $\leq 0.15$ & $\leq 3$ & Very easy \\
II & $1.5-3$ & $2.5-6$ & $2.0-2.4$ & $0.15-0.35$ & $3-6$ & Easy \\
III & $3-6$ & $6-10$ & $2.4-2.75$ & $0.35-0.55$ & $6-9$ & Moderate \\
IV & $6-12$ & $10-18$ & $2.75-3.0$ & $0.55-0.75$ & $9-12$ & Difficult \\
V & $\geq 12$ & $\geq 18$ & $\geq 3.0$ & $\geq 0.75$ & $\geq 12$ & Very difficult \\
\hline
\end{tabular}

density. Therefore, the rock density is generally used as an index for rock mass blastability classification.

2.1.3. Rock Wave Impedance. The dynamic Poisson's ratio, dynamic elastic modulus, bulk modulus, and Lamé parameter for rock can be derived from the P- and S-wave velocities of the rock. All of the physical property indexes of rocks such as the mineral composition, porosity, water-bearing, and weathering degree are captured in the P-wave velocity of the rock. The P-wave velocity of the rock can be easily measured. The rock wave impedance can be obtained by multiplying the P-wave velocity of the rock by the rock density (2). The impedance is a measure of the force of the disturbance required to produce a unit speed of a moving rock particle during the transmission of a stress wave in the rock and is a measure of the resistance of the rock to momentum transfer. Therefore, the rock wave impedance is chosen as one of the indexes for the rock mass blastability classification:

$$
z=\rho v_{\mathrm{pr}}
$$

In the equation above, $z$ denotes the rock wave impedance $\left(10^{6} \mathrm{~kg} / \mathrm{m}^{3} \times \mathrm{m} / \mathrm{s}\right), \rho$ denotes the rock density $\left(\mathrm{kg} / \mathrm{m}^{3}\right)$, and $v_{\text {pr }}$ denotes the P-wave velocity of the rock $(\mathrm{m} / \mathrm{s})$.

2.1.4. Integrity Coefficient of Rock Mass. The geological properties of a rock mass, such as the integrity, fissure, and degree of development of a joint fissure, are captured in the Pwave velocity of the rock mass. A fast wave propagation velocity in a rock mass typically corresponds to mild rock densification, hardness, integrity, and weathering. In contrast, a slow wave propagation velocity corresponds to severe rock porosity, weakness, fragmentation, structural development, and weathering. The integrity coefficient of a rock mass is given by the square of the ratio of the P-wave velocity of a rock mass to the P-wave velocity of the rock (3), which reflects the extent of fracturing for a geological discontinuity, such as a joint fissure. A rock mass with a small integrity coefficient is susceptible to a large amount of rock mass crushing, and the rock mass can be easily blasted. Therefore, the integrity coefficient of the rock mass is chosen as one of the indexes for the rock mass blastability classification:

$$
K_{V}=\left(\frac{v_{\mathrm{pm}}}{v_{\mathrm{pr}}}\right)^{2} .
$$

In the equation above, $K_{V}$ denotes the integrity coefficient of the rock mass, $v_{\mathrm{pm}}$ denotes the P-wave velocity of the rock

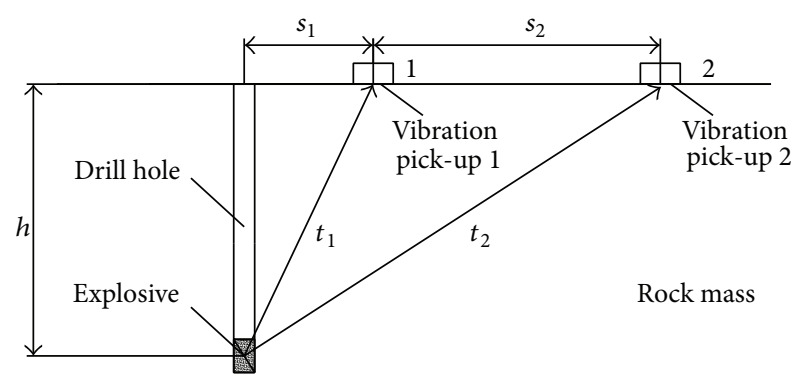

FIGURE 1: Schematic for testing the P-wave velocity of the rock mass.

mass $(\mathrm{m} / \mathrm{s})$, and $v_{\text {pr }}$ denotes the P-wave velocity of the rock $(\mathrm{m} / \mathrm{s})$.

In conclusion, five indexes were chosen for the rock mass blastability classification: the Protodyakonov coefficient and tensile strength of the rock, rock density, rock wave impedance, and the integrity coefficient of the rock mass. Among these indexes, the Protodyakonov coefficient and tensile strength of the rock are mechanical property indexes of the rock, the rock density and rock wave impedance are physical property indexes of the rock; and the integrity coefficient of the rock mass is a measure of the geological properties of the rock mass. These five indexes primarily reflect the relevant physical and mechanical properties and characteristics of the geological structure of a rock mass and blasting and can be easily obtained by field measurements and experiments.

2.2. Determination of the Standards for Rock Mass Blastability Classification. The value selection and designation of classification standards play an important role in the development of models for rock mass blastability classification. In the literature, the standards for classification indexes are determined using five ranks for rock mass blastability: very easy, easy, moderate, difficult, and very difficult. These classification standards for rock mass blastability are shown in Table 1 [18].

\subsection{Measurements and Results for the Indexes}

2.3.1. P-Wave Velocity of the Rock Mass. Figure 1 illustrates a hole that is drilled by a geological drilling rig to measure a rock mass. A small quantity of explosive is placed at the bottom of the hole, and two vibration pick-up instruments are placed around the hole. These two instruments should be 


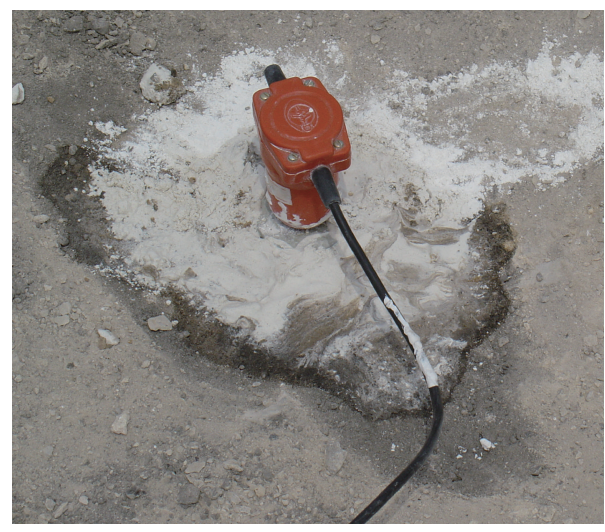

FIGURE 2: Vibration pick-up.

placed as far apart as possible and in a straight line with the hole. Detonating the explosive in the hole produces a surface wave and volume wave in the rock mass, where the volume wave consists of $\mathrm{P}$-wave and S-wave components. The velocity of the P-wave is higher than that of the S-wave, and, thus, the $\mathrm{P}$-wave reaches the measuring point before the $\mathrm{S}$-wave. When the $\mathrm{P}$-wave reaches measuring point 1 , it triggers the "vibration pick-up 1 ," which records the time $t_{1}$ at which the P-wave reaches point 1 . The "vibration pick-up 2" records the time $t_{2}$ at which the $\mathrm{P}$-wave reaches measuring point 2. The P-wave velocity of the rock mass is calculated using the difference between the times that the $\mathrm{P}$-wave reaches measuring points 1 and 2 and the distances between the two measuring points and the center of the explosive cartridge. Equation (4) shows the formula used to calculate the P-wave velocity of the rock mass for the geometry shown in Figure 1 [19]:

$$
v_{\mathrm{pm}}=\frac{\sqrt{h^{2}+\left(s_{1}+s_{2}\right)^{2}}-\sqrt{h^{2}+s_{1}^{2}}}{t_{2}-t_{1}} .
$$

In the equation above, $h$ denotes the depth of the hole (in $\mathrm{m}), s_{1}$ denotes the distance between measuring point 1 and the hole (in $\mathrm{m}$ ), $s_{2}$ denotes the distance between the two points (in $\mathrm{m}$ ), $t_{1}$ denotes the time at which the $\mathrm{P}$-wave reaches point 1 (in $\mathrm{s}$ ), and $t_{2}$ denotes the time at which the $\mathrm{P}$-wave reaches point 2 (in s).

Following the aforementioned principles, field tests were performed at different locations in the Heidaigou open-cast coal mine, where the drilling hole diameter was $127 \mathrm{~mm}$ and the hole depth was between 5 and $17 \mathrm{~m}$. A vibration velocity transducer (model CD-21, manufactured by Beijing Instrument Industry Group Co., Ltd., in Beijing, China) was used as the vibration pick-up instrument, and a blasting vibration instrument (model EXP3850, Chengdu VIDTS Dynamic Instrument Co., Ltd., in Chengdu, China) was used as the blasting vibration recording instrument, as shown in Figures 2 and 3, respectively.

The bench consisted primarily of medium sandstone, and a hole was drilled in the bench at an altitude of $1,185 \mathrm{~m}$, as shown in Figure 4. The depth of the hole was $16.3 \mathrm{~m}$, and the distances $s_{1}$ and $s_{2}$ were 7.1 and $16.7 \mathrm{~m}$, respectively.

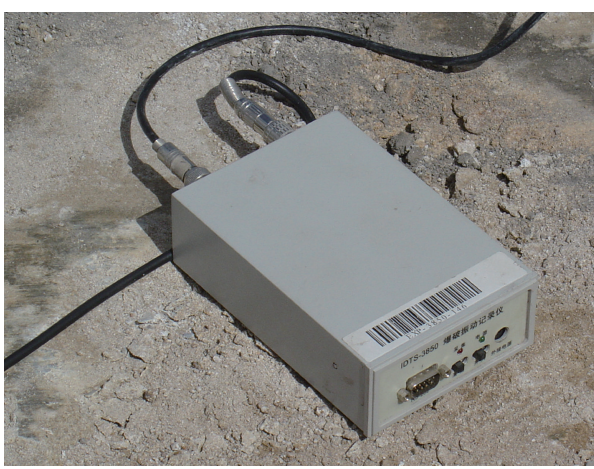

FIGURE 3: Blasting vibration recorder.

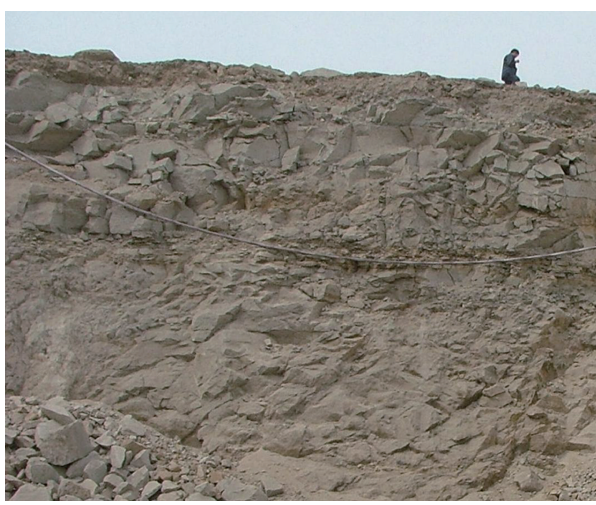

FIgURE 4: Photograph of the rock mass.

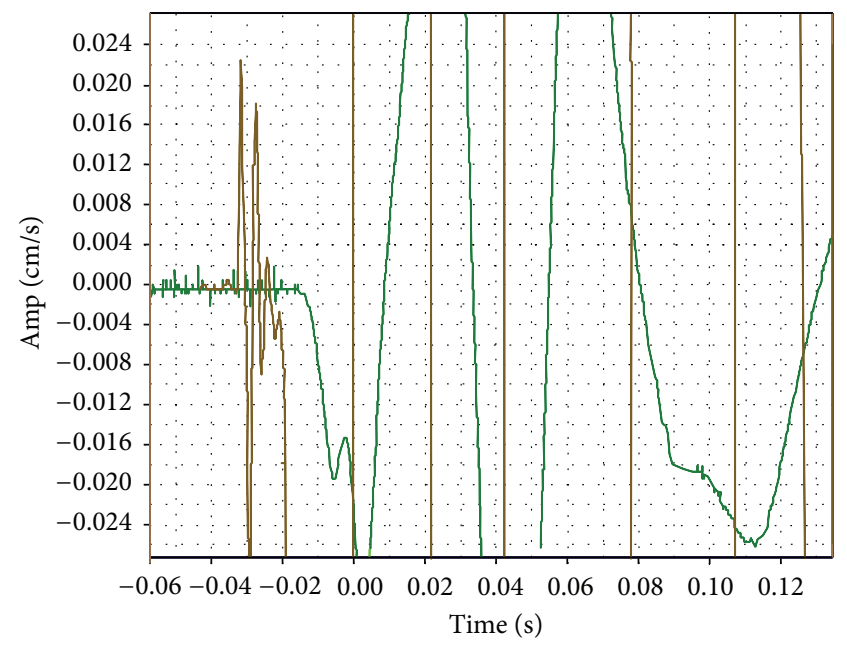

Figure 5: Test pattern of the P-wave velocity of the rock mass.

The two times that were obtained from the test, $t_{1}=$ $-23.65 \mathrm{~ms}$ and $t_{2}=-13.75 \mathrm{~ms}$, are shown in Figure 5. The Pwave velocity of the medium sandstone mass was calculated to be $\mathrm{vpm}=1,118 \mathrm{~m} / \mathrm{s}$ using (4).

Similarly, the measurements indicated that the wave velocity in the mudstone mass was $1,273 \mathrm{~m} / \mathrm{s}$, the wave velocity in the sandstone mass was $944 \mathrm{~m} / \mathrm{s}$, and the wave 


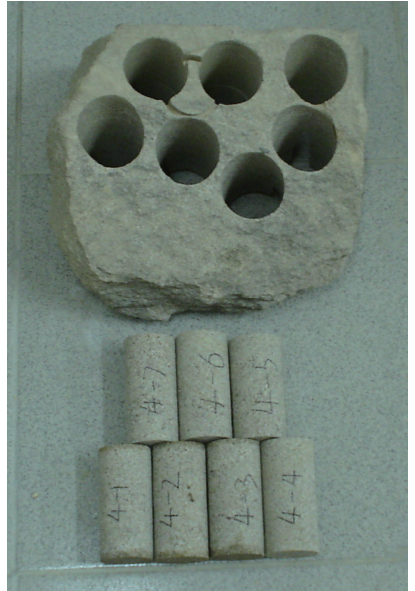

FIGURE 6: Rock samples.

TABLE 2: Test results for the rock mass blastability indexes.

\begin{tabular}{lccccc}
\hline Rock mass & $\begin{array}{c}\sigma_{t} \\
(\mathrm{MPa})\end{array}$ & $\begin{array}{c}\sigma_{p} \\
(\mathrm{MPa})\end{array}$ & $\begin{array}{c}\rho \\
\left(\mathrm{t} / \mathrm{m}^{3}\right)\end{array}$ & $\begin{array}{c}\sigma_{\mathrm{pm}} \\
(\mathrm{m} / \mathrm{s})\end{array}$ & $\begin{array}{c}v_{\mathrm{pr}} \\
(\mathrm{m} / \mathrm{s})\end{array}$ \\
\hline Mudstone & 2.19 & 27.6 & 2.68 & 1273 & 3744 \\
Fine sandstone & 2.72 & 43.6 & 2.41 & 957 & 3226 \\
Medium sandstone & 2.26 & 27.3 & 2.36 & 1118 & 2724 \\
Coarse sandstone & 1.50 & 24.3 & 2.23 & 796 & 2360 \\
\hline
\end{tabular}

velocity in the coarse sandstone rock mass was $796 \mathrm{~m} / \mathrm{s}$ in the Heidaigou open-cast coal mine.

2.3.2. P-Wave Velocity of the Rock Mass. Different types of rocks were collected from the Heidaigou open-cast coal mine and processed into standard samples with specifications of $\phi 50 \mathrm{~mm} \times 100 \mathrm{~mm}$, as shown in Figure 6. A TICO ultrasonic detector was used to determine the P-wave velocity of the rock. The TICO detector had two sensors, a sender and a receiver. The distance 1 between the two sensors and the propagation time $t$ of the test sound wave to travel between the two sensors were used to calculate the wave velocity $v_{\mathrm{pr}}=$ $l / t$. The wave velocities were as follows: $3,744 \mathrm{~m} / \mathrm{s}$ for the mudstone, $3,226 \mathrm{~m} / \mathrm{s}$ for the fine sandstone, $2,724 \mathrm{~m} / \mathrm{s}$ for the medium sandstone, and 2,360 m/s for the coarse sandstone.

2.3.3. Rock Density, Tensile Strength, and Compressive Strength. The rock density was determined by weighing the samples and calculating the sample volumes.

The tensile strength and uniaxial compressive strength of the rock were measured using microcomputer control electron universal testing machines (model WDW300, KeXin Testing Machine Co., Ltd., in Changchun, China). The specifications for the test samples for the uniaxial compressive strength measurements were $\phi 50 \mathrm{~mm} \times 100 \mathrm{~mm}$. The Brazil splitting method was used to measure the tensile strength of the rock with specifications of $\phi 50 \mathrm{~mm} \times 25 \mathrm{~mm}$.

The test results for the rock density, rock tensile strength, and rock compressive strength are provided in Table 2.
TABLE 3: Rock mass blastability classification index values.

\begin{tabular}{lccccc}
\hline Rock mass & $\begin{array}{c}\sigma_{t} \\
(\mathrm{MPa})\end{array}$ & $f$ & $\begin{array}{c}P \\
\left(\mathrm{t} / \mathrm{m}^{3}\right)\end{array}$ & $K_{v}$ & $\begin{array}{c}Z \\
\left(10^{6} \mathrm{~kg} / \mathrm{m}^{3} \times \mathrm{m} / \mathrm{s}\right)\end{array}$ \\
\hline Mudstone & 2.19 & 2.76 & 2.68 & 0.12 & 10.03 \\
Fine sandstone & 2.72 & 4.36 & 2.41 & 0.09 & 7.77 \\
Medium sandstone & 2.26 & 2.73 & 2.36 & 0.17 & 6.43 \\
Coarse sandstone & 1.50 & 2.43 & 2.23 & 0.11 & 5.26 \\
\hline
\end{tabular}

TABLE 4: Judgment matrix $P$.

\begin{tabular}{lccccc}
\hline Variables & $\sigma_{t}$ & $f$ & $\rho$ & $K_{v}$ & $z$ \\
\hline$\sigma_{t}$ & 1 & $1 / 5$ & $1 / 3$ & $1 / 4$ & $1 / 2$ \\
$f$ & 5 & 1 & 3 & 2 & 4 \\
$P$ & 3 & $1 / 3$ & 1 & $1 / 2$ & 2 \\
$K_{v}$ & 4 & $1 / 2$ & 2 & 1 & 3 \\
$z$ & 2 & $1 / 4$ & $1 / 2$ & $1 / 3$ & 1 \\
\hline
\end{tabular}

The classification index values of the rock masses were calculated and are shown in Table 3.

\section{Determination of the Index Weights Using the Combination Weight Method}

To circumvent the deficiencies of the subjective weight method and the objective weight method, the index weight was systematically determined using the analysis hierarchy process (AHP) to calculate the subjective weight, the entropy method (EM) was used to calculate the objective weight, and the combination weight method (CWM), which is based on game theory (GT), was used to determine the index weights for the rock mass blastability classification.

3.1. Determination of Subjective Weight Using the Analysis Hierarchy Process. AHP is a systematic, hierarchical analysis method that incorporates both qualitative and quantitative analyses; thus, AHP is simple and practical and avoids the uncertainties and errors that can arise when determining the index weights. The methodology used to calculate the weights using the AHP is detailed in Saaty's papers [20, 21].

A pairwise comparison of the Protodyakonov coefficient, tensile strength, density, wave impedance, and integrity coefficient of the rock mass was performed to construct a judgment matrix, as shown in Table 4.

The calculation produced the eigenvector $\mathbf{W}=(0.06$, $0.42,0.16,0.26,0.10)^{\mathrm{T}}$, maximum eigenvalue $\lambda_{\max }=5.068$, and the consistency index $\mathrm{CI}=0.017$. For $n=5$ elements, the random index was found to be $\mathrm{RI}=1.12$, which was used to calculate the consistency ratio $\mathrm{CR}=0.017 / 1.12=0.015<$ 0.1 . Therefore, the judgment matrix satisfied the consistency check. The consistency ratio weight vector was determined using AHP to be $\mathbf{W}_{1}=(0.06,0.42,0.16,0.26,0.10)$.

3.2. Determination of the Objective Weight Using the Entropy Method. Information theory states that the information entropy is a measure of the degree of disorder in a system. 
Lower information entropy indicates a lower degree of disorder and a higher utility value of the information; the opposite holds true for higher information entropy [22]. An indicator that does not produce different effects for different levels of rock mass blastability is not useful for rock mass blastability classification. Thus, if an indicator produces a smaller difference for different levels of rock mass blastability, the effect of the indicator on the rock mass blastability classification is smaller, and the corresponding information entropy is higher; the opposite holds true for an indicator that produces a larger difference for different levels of rock mass blastability. That is, the difference degree of the indicator for the rock mass blastability classification is inversely proportional to the information entropy. Therefore, the index weight was determined from the difference degree of the rock mass blastability classification indicator using the EM. The calculation procedure is detailed in the papers of PeiYue et al. [23] and Zou et al. [24].

Using the sample data for mudstone, fine sandstone, medium sandstone, and coarse sandstone that are given in Table 3, the entropy of each indicator was calculated to be $\mathbf{H}=(0.984$ 8, 0.979 6, 0.998 4, 0.979 9, 0.979 5), which yielded the consistency ratio weight vector $\mathbf{W}_{2}=(0.20,0.26$, $0.02,0.26,0.26)$.

3.3. Combination Weight from Game Theory. The weights obtained from different weight methods may not be consistent with each other. GT can be used to minimize the sum of the differences between the final determined weight and the weight determined from each method [25]. The procedure for determining the GT-based combination weight is given below [26].

Assume that $L$ methods are used to weight the indexes of the rock mass blastability classification, where the $L$ weight vectors are given by $\mathbf{W}_{k}=\left(\omega_{k 1}, \omega_{k 2}, \ldots, \omega_{k n}\right)$ (where $1 \leq k \leq$ $L$, and $n$ indicates the number of indexes of the rock mass blastability classification). A combination weight vector can be constructed from a random linear combination of the $L$ weight vectors as follows:

$$
\mathbf{W}_{c}=\sum_{k=1}^{L} \alpha_{k} \cdot \mathbf{W}_{k}^{\mathrm{T}}
$$

In the equation above, $\mathbf{W}_{c}$ denotes the combination weight vector and $\alpha_{k}$ denotes the coefficient of the linear combination, where $\alpha_{k}>0$.

The most satisfactory combination weight vector is obtained by minimizing the deviation between the combination weight vector $\mathbf{W}_{c}$ and each weight vector $\mathbf{W}_{k}$ by optimizing the coefficient $\alpha_{k}$ of the $L$ linear combinations. The game model is given as

$$
\min \left\|\sum_{k=1}^{L} \alpha_{k} \cdot \mathbf{W}_{k}^{\mathrm{T}}-\mathbf{W}_{l}^{\mathrm{T}}\right\|_{2} \quad(l=1,2, \ldots, L) .
$$

The differential attribute of the matrix is used to calculate the optimum first derivative condition in (6), which can be written in the following matrix form:

$$
\begin{gathered}
{\left[\begin{array}{cccc}
\mathbf{W}_{1} \cdot \mathbf{W}_{1}^{\mathrm{T}} & \mathbf{W}_{1} \cdot \mathbf{W}_{2}^{\mathrm{T}} & \cdots & \mathbf{W}_{1} \cdot \mathbf{W}_{L}^{\mathrm{T}} \\
\mathbf{W}_{2} \cdot \mathbf{W}_{1}^{\mathrm{T}} & \mathbf{W}_{2} \cdot \mathbf{W}_{2}^{\mathrm{T}} & \cdots & \mathbf{W}_{2} \cdot \mathbf{W}_{L}^{\mathrm{T}} \\
\vdots & \vdots & \ddots & \vdots \\
\mathbf{W}_{L} \cdot \mathbf{W}_{1}^{\mathrm{T}} & \mathbf{W}_{L} \cdot \mathbf{W}_{2}^{\mathrm{T}} & \cdots & \mathbf{W}_{L} \cdot \mathbf{W}_{L}^{\mathrm{T}}
\end{array}\right]\left[\begin{array}{c}
\alpha_{1} \\
\alpha_{2} \\
\vdots \\
\alpha_{L}
\end{array}\right]} \\
=\left[\begin{array}{c}
\mathbf{W}_{1} \cdot \mathbf{W}_{1}^{\mathrm{T}} \\
\mathbf{W}_{2} \cdot \mathbf{W}_{2}^{\mathrm{T}} \\
\vdots \\
\mathbf{W}_{L} \cdot \mathbf{W}_{L}^{\mathrm{T}}
\end{array}\right] .
\end{gathered}
$$

The matrix is used to calculate the normalization processing of $\left(\alpha_{1}, \alpha_{2}, \ldots, \alpha_{L}\right)$, as shown in (8), to obtain the optimal linear combining coefficient $\alpha_{k}^{*}(1 \leq k \leq L)$ as follows:

$$
\alpha_{k}^{*}=\frac{\alpha_{k}}{\sum_{k=1}^{L} \alpha_{k}} \text {. }
$$

Therefore, the optimal combination weight vector is

$$
\mathbf{W}^{*}=\sum_{k=1}^{L} \alpha_{k}^{*} \cdot \mathbf{W}_{k} \text {. }
$$

In the equation above, $\mathbf{W}^{*}$ denotes the optimal combination weight vector, $\mathbf{W}^{*}=\left(\omega_{j}^{*}\right)_{1 \times n}$, where $\omega_{j}^{*}$ is the optimal combination weight vector for the $j$ th index, with $\sum_{i=1}^{n} \omega_{j}^{*}=$ 1.

The calculated subjective and objective weights were used to determine $\alpha_{1}^{*}=0.6912$ and $\alpha_{2}^{*}=0.3088$ using (7) and $(8)$, and $\mathbf{W}^{*}=(0.10,0.37,0.12,0.26,0.15)$ was calculated using (9). The index weights that were determined using the AHP, EM, and CWM are shown in Figure 7.

Figure 7 illustrates that the index weights that were determined using the combination weight method lay between those calculated using the AHP and those calculated using EM. The CWM balanced and coordinated the impacts that the subjective and objective methods exerted on the weights, thus overcoming the one-sidedness of each method; the finalized CWM produced more realistic results than the individual methods.

\section{Fuzzy Pattern Recognition Model for Rock Mass Blastability}

4.1. Model Principles. We assume that the rock mass blastability can be divided into $m$ levels for $n$ indexes, where each blastability has its own grading standards (the value range of each index) and that the $m$ grading standards of the rock mass blastability can serve as the $m$ fuzzy subsets $\widetilde{A}_{1}, \widetilde{A}_{2}, \ldots, \widetilde{A}_{m}$ that constitute a standard sample database $\left\{\widetilde{A}_{1}, \widetilde{A}_{2}, \ldots, \widetilde{A}_{m}\right\}$. The value of the $j$ th grading standard of the rock mass 


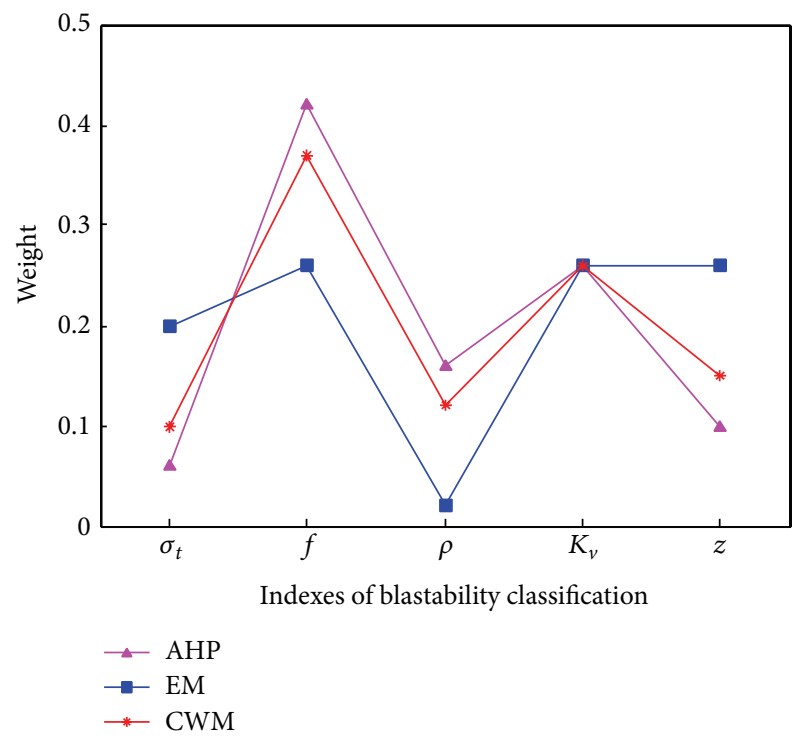

FIGURE 7: Comparison of weights.

blastability classification is denoted by $x_{j}(j=1,2, \ldots, n)$, and the universe $U=\left\{u \mid u=\left(x_{1}, x_{2}, \ldots, x_{n}\right)\right\}$, namely, $u$, denotes the set of $n$ indexes for a certain rock mass sample.

The membership functions $\mu_{i}\left(x_{j}\right)$ and $\mu_{i}(u)$ are constructed for a particular fuzzy subset $\widetilde{A}_{i}$. For these functions, $i(i=1,2, \ldots, m)$ denotes the number of the rock mass blastability classification for a standard sample, $j(j=$ $1,2, \ldots, n)$ denotes the index number, and $x_{j}$ indicates the value of the $j$ th index for a specific rock mass sample. $\mu_{i}\left(x_{j}\right)$ denotes the membership degree of the $j$ th index of a specific rock mass sample that relatively belongs to the blastability degree of the $i$ th rock mass, and $\mu_{i}(u)$ is the membership degree of a specific rock mass sample that relatively belongs to the blastability degree of the $i$ th rock mass.

If $u_{0} \in U$ and $k \in\{1,2, \ldots, m\}$ exist such that $\mu_{k}\left(u_{0}\right)=$ $\max \left\{\mu_{1}\left(u_{0}\right), \mu_{2}\left(u_{0}\right), \ldots, u_{m}\left(x_{0}\right)\right\}, u_{0}$ is considered to belong to $\widetilde{A}_{k}$, which means that the blastability degree of rock mass sample $u_{0}$ belongs to degree $k$.

4.2. Development of the Membership Function. To establish a fuzzy relation between the classification indexes and the standard samples, the membership functions must first be developed between each index and each standard sample [27]. The fuzziest principle and clearest principle must be obeyed while formulating the membership function. That is, the membership degree is 0.5 at the endpoint of the interval for the fuzziest state, and the membership degree is 1 at the midpoint of the interval for the clearest state. Moreover, the sum of the membership degrees at any point is 1 [28].

Commonly used membership functions include triangular, trapezoidal, normal distribution and mountain-shaped membership functions [29]. Mikkili and Panda concluded that there are no considerable differences among the membership degrees that correspond to different membership
TABLE 5: Value range of the index.

\begin{tabular}{lccccc}
\hline $\begin{array}{l}\text { Index } \\
\text { number }\end{array}$ & Degree 1 & Degree 2 & $\cdots$ & Degree $m-1$ & Degree $m$ \\
\hline$j$ & $\leq a_{1 j}$ & $a_{1 j} \sim a_{2 j}$ & $\cdots$ & $a_{(m-2) j} \sim a_{(m-1) j}$ & $\geq a_{(m-1) j}$ \\
\hline
\end{tabular}

functions, and the analysis results are consistent [30]. Therefore, a trapezoidal membership function was used to formulate the fuzzy assessment matrix. Based on the principle used to identify the membership function, the trapezoidal membership function degenerated into a triangular membership function.

Table 1 illustrates that the values of the classification indexes that were chosen in this study increased with the degree of rock mass blastability, namely, the incremental index. The assumed value range of the $j$ th index for the $i$ th degree is provided in Table 5.

Let us consider the trapezoidal membership function as an example, where the membership functions for each index of each degree are given as follows:

$$
\begin{gathered}
\mu_{1}\left(x_{j}\right)= \begin{cases}1 & x_{j}<b_{1 j} \\
\frac{x_{j}+b_{1 j}-2 a_{1 j}}{2\left(b_{1 j}-a_{1 j}\right)} & b_{1 j} \leq x_{j}<a_{1 j} \\
\frac{b_{2 j}-x_{j}}{2\left(b_{2 j}-a_{1 j}\right)} & a_{1 j} \leq x_{j}<b_{2 j} \\
0 & x_{j} \geq b_{2 j},\end{cases} \\
\mu_{i}\left(x_{j}\right) \begin{cases}0 & x_{j}<b_{(i-1) j}, x_{j} \geq b_{(i+1) j} \\
\frac{0}{2\left(a_{(i-1) j}-b_{(i-1) j}\right)} & b_{(i-1) j} \leq x_{j}<a_{(i-1) j} \\
\frac{x_{j}+b_{i j}-2 a_{(i-1) j}}{2\left(b_{i j}-a_{(i-1) j}\right)} & a_{(i-1) j} \leq x_{j}<b_{i j} \\
\frac{b_{i j}-2 a_{i j}-x_{j}}{2\left(a_{i j}-b_{i j}\right)} & b_{i j} \leq x_{j}<a_{i j} \\
\frac{b_{(i+1) j}-x_{j}}{2\left(b_{(i+1) j}-a_{i j}\right)} & a_{i j} \leq x_{j}<b_{(i+1) j}, \\
\left.x_{j}\right)= \begin{cases}0 & x_{j}-b_{(m-1) j} \\
b_{m j}-b_{(m-1) j} & x_{j}<b_{(m-1) j}\end{cases} \end{cases} \\
\quad x_{j-1) j} \leq x_{j}<b_{m j} .
\end{gathered}
$$

In the equations above, $x_{j}$ denotes the value of the $j$ th index for a given rock sample, $\mu_{i}\left(x_{j}\right)$ denotes the membership degree of index $x_{j}$ that relatively belongs to the $i$ th blastability degree, and $b_{m j}=2 a_{(m-1) j}-b_{(m-1) j}$, where $b_{1 j}=\max \left\{2 a_{1 j}-\right.$ $\left.b_{2 j}, a_{1 j} / 2\right\}$, and $b_{i j}=\left(a_{(i-1) j}+a_{i j}\right) / 2(2 \leq i \leq m-1)$. 


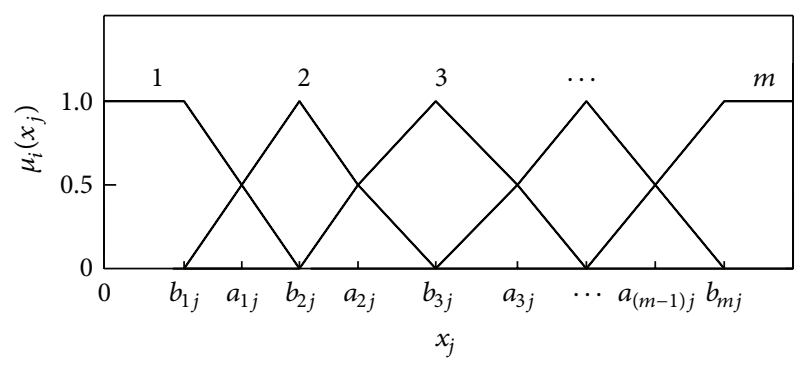

FIGURE 8: Pictorial representation of trapezoidal membership functions.

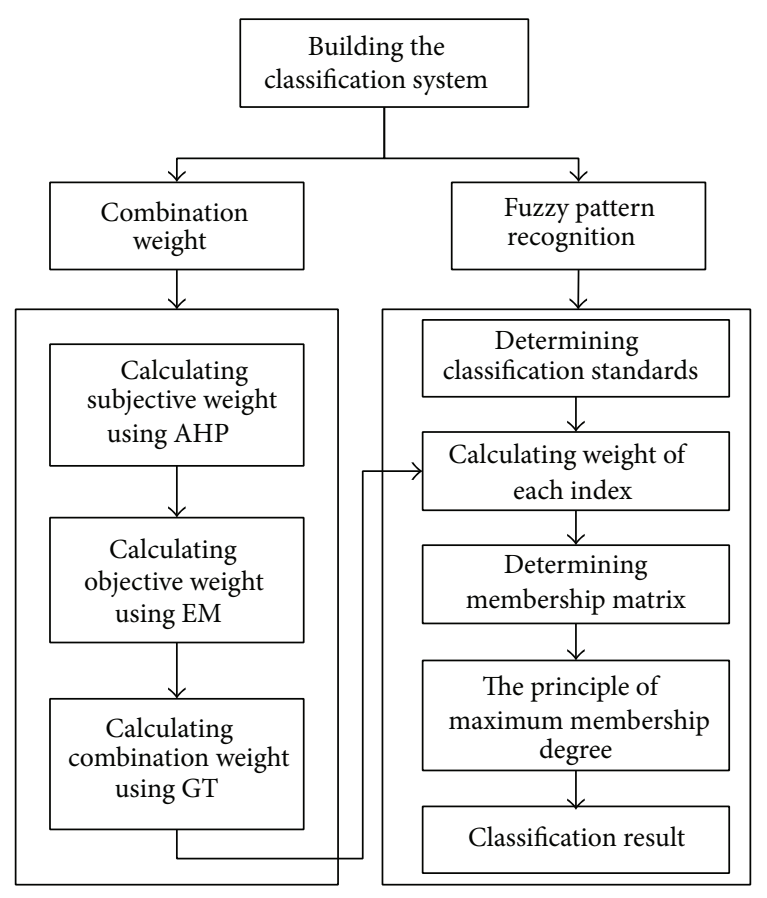

FIGURE 9: Rock mass blastability classification procedure.

Equations (10) correspond to the membership function of the incremental index, which is shown in Figure 8. The membership function of the descending index can be determined using the same method and is thus not presented here.

4.3. Identification of the Rock Mass Blastability Degree. The procedure for the rock mass blastability classification is summarized in Figure 9. The membership degree of every blastability degree can be calculated using (11) together with the classification index and the classification standard provided in section two, the combination weight provided in section three, and the membership function provided in section four for any type of rock as long as the values of the $\mathrm{n}$ indexes are known, and the blastability degree of the rock can be determined using the principle of the maximum membership degree. Consider

$$
\mu_{i}(u)=\frac{1}{n} \sum_{j=1}^{n} \omega_{j}^{*} \mu_{i}\left(x_{j}\right) \quad 1 \leq i \leq m .
$$

TABLE 6: Membership degree for each index for mudstone.

\begin{tabular}{lccccc}
\hline Blastability class & $\sigma_{t}$ & $f$ & $\rho$ & $K_{v}$ & $z$ \\
\hline I & 0.04 & 0.43 & 0 & 0.73 & 0 \\
II & 0.96 & 0.57 & 0 & 0.27 & 0 \\
III & 0 & 0 & 0.70 & 0 & 0.16 \\
IV & 0 & 0 & 0.30 & 0 & 0.84 \\
V & 0 & 0 & 0 & 0 & 0 \\
\hline
\end{tabular}

TABLE 7: Membership degree of each rock mass.

\begin{tabular}{lccccc}
\hline \multirow{2}{*}{ Rock mass } & \multicolumn{5}{c}{ Blastability class } \\
& I & II & III & IV & V \\
\hline Mudstone & 0.35 & 0.38 & 0.11 & 0.16 & 0.00 \\
Fine sandstone & 0.24 & 0.51 & 0.24 & 0.01 & 0.00 \\
Medium sandstone & 0.27 & 0.59 & 0.14 & 0.00 & 0.00 \\
Coarse sandstone & 0.44 & 0.51 & 0.05 & 0.00 & 0.00 \\
\hline
\end{tabular}

In the equation above, $\omega_{j}^{*}$ denotes the satisfactory combination weight of the $j$ th index.

\section{Project Application}

5.1. Rock Mass Blastability Classification. The identified classification index provided in Table 1 was used to calculate the specific membership function using (10). Let us consider the rock mass blastability classification of mudstone from the Heidaigou open-cast coal mine as an example. The membership degree between each index and each rock mass blastability classification was calculated by substituting the relevant indexes of the rock mass of mudstone into the membership function in Table 3 and is shown in Table 6.

Equation (11) was used to calculate the following values: $\mu_{1}(u)=0.35, \mu_{2}(u)=0.38, \mu_{3}(u)=0.11, \mu_{4}(u)=0.16$, and $\mu_{5}(u)=0$. The principle of the maximum membership degree was used to determine that the largest rock mass blastability degree of mudstone was $\mu_{2}(u)$, which corresponds to a degree of 2 , the "easy" blasted rock mass.

The membership degree between each index for the rock masses of fine sandstone, medium sandstone, and coarse sandstone and the rock mass blastability classification was similarly confirmed, and the membership degree between each rock mass and the rock mass blastability classification was calculated, as shown in Table 7 and Figure 10. The principle of the maximum membership degree indicated that the rock masses of fine sandstone, medium sandstone, and coarse sandstone could all be categorized as easy blasted rock masses.

Figure 10 illustrates that although each rock mass had a maximum membership degree for a blastability of II, which belonged to the easy blasted rock mass, the mudstone and coarse sandstone rock mass also had a large membership degree for a blastability of I, indicating that the degree for mudstone and coarse sandstone was between the very easy blasted and easy blasted rock masses. Although the fine sandstone and coarse sandstone had equal membership degrees for a blastability of II, the coarse sandstone had a 


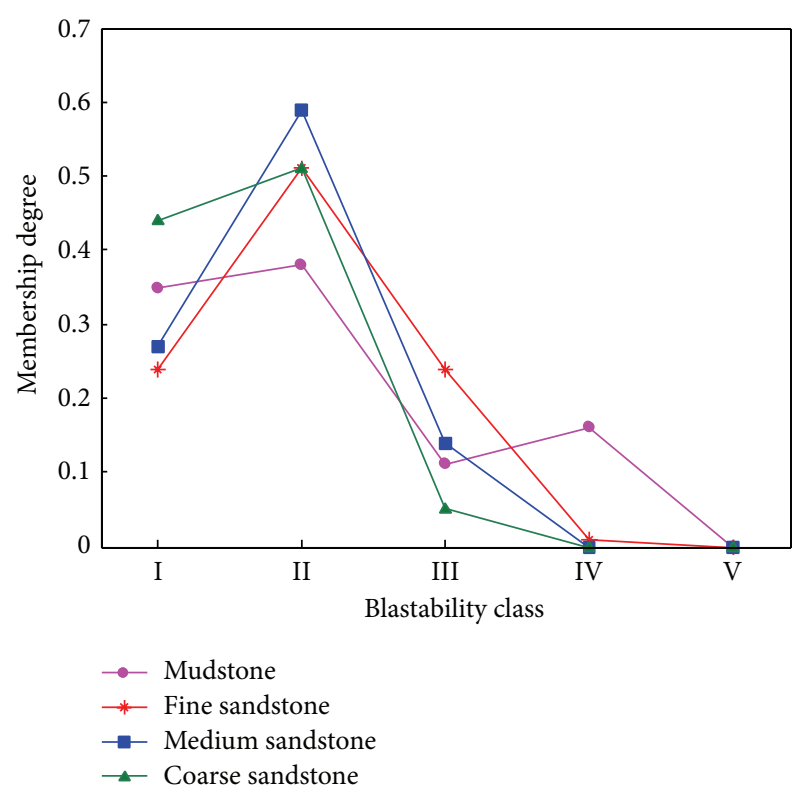

FIGURE 10: Membership degree of each rock mass.

TABLE 8: Unit explosive consumption of rock mass of each classification.

\begin{tabular}{lc}
\hline Blastability class & Unit explosive consumption $\left(\mathrm{kg} / \mathrm{m}^{3}\right)$ \\
\hline I & $\leq 0.35$ \\
II & $0.35-0.45$ \\
III & $0.45-0.65$ \\
IV & $0.65-0.90$ \\
V & $\geq 0.90$ \\
\hline
\end{tabular}

larger membership degree for a blastability of I and a smaller membership degree for a blastability of III, illustrating that coarse sandstone was more explosive than fine sandstone despite both sandstones being categorized as easy blasted rock mass.

5.2. Unit Explosive Consumption. The relevant literature and material and blasting experience from open-cast mines was used to determine the unit explosive consumption (ANFO) for the loose blasting of a rock mass for each classification, as shown in Table 8.

Let $q_{2}, q_{3}$, and $q_{4}$ denote the midpoints of the interval of the unit explosive consumption for degrees II, III, and IV in Table 8, respectively, where $q_{1}=0.35-\left(q_{2}-0.35\right)$ and $q_{5}=0.90+\left(0.90-q_{4}\right)$ form the vector $Q=\left(q_{i}\right)_{1 \times 5}=$ $(0.30,0.40,0.55,0.78,1.02)$.

The membership matrix $\mathbf{U}=\left(\mu_{i}(u)\right)_{1 \times 5}$ between the rock mass and the rock mass blastability degree was calculated. The unit explosive consumption for the loose blasting of the rock mass was then calculated using

$$
q= \begin{cases}\leq 0.3 & \mu_{1}(u)=1 \\ \geq 1.02 & \mu_{5}(u)=1 \\ Q \cdot U^{\mathrm{T}} & \mu_{1}(u) \neq 1, \mu_{5}(u) \neq 1 .\end{cases}
$$

Consider the mudstone rock mass in the Heidaigou open-cast coal mine as an example, where the unit explosive consumption for loose blasting was calculated to be $q_{\mathrm{mud}}=$ $(0.30,0.40,0.55,0.78,1.02) \cdot(0.35,0.38,0.11,0.16,0)=$ $0.44 \mathrm{~kg} / \mathrm{m}^{3}$. Similarly, the unit explosive consumption values for fine sandstone, medium sandstone, and coarse sandstone were determined to be $q_{f}=0.42 \mathrm{~kg} / \mathrm{m}^{3}, q_{\text {med }}=0.40 \mathrm{~kg} / \mathrm{m}^{3}$, and $q_{c}=0.36 \mathrm{~kg} / \mathrm{m}^{3}$, respectively.

When there are several types of rock masses in a blasting area, the unit explosive consumption can be approximated using

$$
q_{a}=\frac{\sum_{i=1}^{n} q_{m i} V_{m i}}{\sum_{i=1}^{n} V_{m i}} .
$$

In the equation above, $q_{a}$ denotes the average unit explosive consumption (in $\mathrm{kg} / \mathrm{m}^{3}$ ) for loose blasting in the blasting area, $q_{m i}$ denotes the unit explosive consumption (in $\mathrm{kg} / \mathrm{m}^{3}$ ) for loose blasting of the rock mass $i, V_{m i}$ denotes the volume (in $\mathrm{m}^{3}$ ) of the rock mass $i$, and $n$ denotes the number of types of rock masses in the blasting area.

When there are several rock masses that are approximately level or when there is a gentle incline from the top to the bottom of the blasting area, $V_{m i}$ can be replaced by $h_{m i}$ in (13); thus, $h_{m i}$ denotes the thickness of the rock mass $i(\mathrm{~m})$.

\section{Conclusions and Further Research}

(1) There are no clear boundaries between different classifications for the blastability of a rock mass; that is, there is fuzziness in the classification problem. Thus, a fuzzy pattern recognition method was used to develop a model for rock mass blastability classification. The classification results from the model were obtained as a vector, which provided more information than a point value. Thus, a simple algorithm was used to make full use of the information, and the classification procedure was easy to understand.

(2) The relevant indexes of each rock mass that was tested in the Heidaigou open-cast coal mine were used with the developed rock mass blastability classification model to demonstrate that mudstone, fine sandstone, medium sandstone, and coarse sandstone in the Heidaigou open-cast coal mine could all be considered easy blasted rock mass. The unit explosive consumption of mudstone, fine sandstone, medium sandstone, and coarse sandstone was determined to be $0.44,0.42,0.40$, and $0.36 \mathrm{~kg} / \mathrm{m}^{3}$, respectively. Blasting experiments in the Heidaigou open-cast coal mine demonstrated the accuracy of the aforementioned conclusions. These conclusions were used to develop a loose blasting design, which yielded good results.

(3) The standards for the blastability classification of the rock masses were determined from the literature. These standards can be applied to actual situations but are not based on sufficiently large amounts of sample data; therefore, the scientific basis of these standards has not been confirmed. In addition, a 
sufficiently large amount of sample data was not used with the objective weight method. Thus, this insufficient sample size likely affected the weights that were determined by the objective weight method to some extent. Therefore, the next step is to collect a large amount of sample data of rock masses in typical areas for use in a more accurate calculation of the weight of each classification index and a more accurate calculation and analysis of the standards for the blastability classification of rock masses.

\section{Conflict of Interests}

The authors declare that there is no conflict of interests regarding the publication of this paper.

\section{Acknowledgment}

Financial support for this work, provided by the National High Technology Research and Development Program of China (no. 2012AA062002), is gratefully acknowledged.

\section{References}

[1] J. Zhou and X. Li, "Integrating unascertained measurement and information entropy theory to assess blastability of rock mass," Journal of Central South University, vol. 19, no. 7, pp. 1953-1960, 2012.

[2] Q. Y. Ma and Z. H. Zhang, "Classification of frozen soil blastability by using perception neural network," Journal of Coal Science \& Engineering, vol. 8, no. 1, pp. 54-58, 2002.

[3] J.-L. Shang, J.-H. Hu, R.-S. Mo, X.-W. Luo, and K.-P. Zhou, "Predication model of game theory-matter-element extension for blastability classification and its application," Journal of Mining \& Safety Engineering, vol. 30, no. 1, pp. 86-92, 2013.

[4] J.-P. Latham and P. Lu, "Development of an assessment system for the blastability of rock masses," International Journal of Rock Mechanics and Mining Sciences, vol. 36, no. 1, pp. 41-55, 1999.

[5] S. R. Chen, S. Q. Xie, Y. L. Li, Y. X. Yu, and P. Z. Wu, "Experimental study of physical properties and drillability and blastability of special ore-bodies," Journal of Central South University of Technology, vol. 35, no. 4, pp. 667-669, 2004.

[6] Y. D. Cai and L. S. Yao, "Artificial neural network approach of determining the grade of the blasting classification of rocks," Blasting, vol. 10, no. 2, pp. 50-52, 1993.

[7] X. T. Feng, "A study on neural network on rock blastability," Explosion and Shock Waves, vol. 14, no. 4, pp. 298-306, 1994.

[8] X. T. Feng, "A neural network approach to comprehensive classification of rock stability, blastability and drillability," International Journal of Surface Mining, Reclamation and Environment, vol. 9, no. 2, pp. 57-62, 1995.

[9] C. Fang, X.-G. Zhang, and Z.-H. Dai, "Projection pursuit regression method of rock blastability classification based on artificial fish-swarm algorithm," Blasting, vol. 26, no. 3, pp. 1417, 2009.

[10] Y. D. Cai, "Application of genetic programming in determining the blasting classification of rocks," Explosion and Shock Waves, vol. 15, no. 4, pp. 329-334, 1995.
[11] P. P. Huang, "Rock blastability classification with fuzzy synthesis," Quarterly of the Changsha Institute of Mining Research, vol. 9, no. 4, pp. 63-72, 1989.

[12] Y. Azimi, M. Osanloo, M. Aakbarpour-Shirazi, and A. Aghajani Bazzazi, "Prediction of the blastability designation of rock masses using fuzzy sets," International Journal of Rock Mechanics and Mining Sciences, vol. 47, no. 7, pp. 1126-1140, 2010.

[13] A. Aydin, "Fuzzy set approaches to classification of rock masses," Engineering Geology, vol. 74, no. 3-4, pp. 227-245, 2004.

[14] S. J. Qu, S. L. Mao, W. S. Lu, M. Y. Xin, Y. J. Gong, and X. Y. Jin, "A method for rock mass blastability classifcation based on weighted clustering analysis," Journal of University of Science and Technology Beijing, vol. 28, no. 4, pp. 324-329, 2006.

[15] J.-G. Xue, J. Zhou, X.-Z. Shi, H.-Y. Wang, and H.-Y. Hu, "Assessment of classification for rock mass blastability based on entropy coefficient of attribute recognition model," Journal of Central South University (Science and Technology), vol. 41, no. 1, pp. 251-256, 2010.

[16] Y. L. Yu, D. S. Wang, and S. J. Qu, "Zoning of the blasting compliance of rocks in shuichang open pit," Chinese Journal of Rock Mechanics and Engineering, vol. 9, no. 3, pp. 195-201, 1990.

[17] S.-J. Qu, M.-Y. Xin, S.-L. Mao et al., "Correlation analyses of blastability indexes for rock mass," Chinese Journal of Rock Mechanics and Engineering, vol. 24, no. 3, pp. 468-473, 2005.

[18] P. Lu, The characterisation and analysis of in-situ and blasted block size distribution and the blastability of rock masses [Ph.D. thesis], University of London, London, UK, 1997.

[19] K. M. Li, Z. H. Guo, and Y. Zhang, Research and Application of Casting Blast Technologies in Opencast Mine, China Coal Industry Publishing House, Beijing, China, 2011.

[20] T. L. Saaty, "How to make a decision: the analytic hierarchy process," European Journal of Operational Research, vol. 48, no. 1, pp. 9-26, 1990.

[21] T. L. Saaty, "Decision making with the analytic hierarchy process," International Journal of Services Sciences, vol. 1, no. 1, pp. 83-98, 2008.

[22] J. Wu, J. Sun, L. Liang, and Y. Zha, "Determination of weights for ultimate cross efficiency using Shannon entropy," Expert Systems with Applications, vol. 38, no. 5, pp. 5162-5165, 2011.

[23] L. Pei-Yue, Q. Hui, and W. Jian-Hua, "Application of set pair analysis method based on entropy weight in groundwater quality assessment-a case study in dongsheng city, northwest China," E-Journal of Chemistry, vol. 8, no. 2, pp. 851-858, 2011.

[24] Z. H. Zou, Y. Yun, and J. N. Sun, "Entropy method for determination of weight of evaluating indicators in fuzzy synthetic evaluation for water quality assessment," Journal of Environmental Sciences, vol. 18, no. 5, pp. 1020-1023, 2006.

[25] W. Zhang, J.-P. Chen, Q. Wang et al., "Susceptibility analysis of large-scale debris flows based on combination weighting and extension methods," Natural Hazards, vol. 66, no. 2, pp. 10731100, 2013.

[26] D. C. Chi, T. Ma, and S. Li, "Application of extension assessment method based on game theory to evaluate the running condition of irrigation areas," Transactions of the CSAE, vol. 24, no. 8, pp. 36-39, 2008.

[27] H. Karimnia and H. Bagloo, "Optimum mining method selection using fuzzy analytical hierarchy process-Qapiliq salt mine, Iran," International Journal of Mining Science and Technology, vol. 25, no. 2, pp. 225-230, 2015.

[28] N. A. Bahri, F. M. A. Ebrahimi, and S. G. Reza, "A fuzzy logic model to predict the out-of-seam dilution in longwall mining," 
International Journal of Mining Science and Technology, vol. 25, no. 1, pp. 91-98, 2015.

[29] Y. H. Su, M. C. He, and X. M. Sun, "Equivalent characteristic of membership function type in rock mass fuzzy classification," Journal of University of Science and Technology Beijing, vol. 29, no. 7, pp. 670-675, 2007.

[30] S. Mikkili and A. K. Panda, "Simulation and real-time implementation of shunt active filter id-iq control strategy for mitigation of harmonics with different fuzzy membership functions," IET Power Electronics, vol. 5, no. 9, pp. 1856-1872, 2012. 


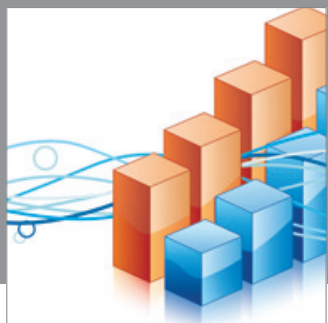

Advances in

Operations Research

mansans

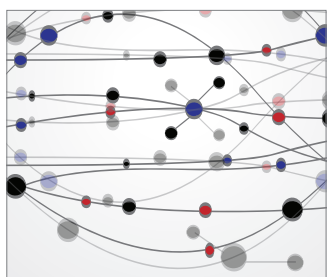

The Scientific World Journal
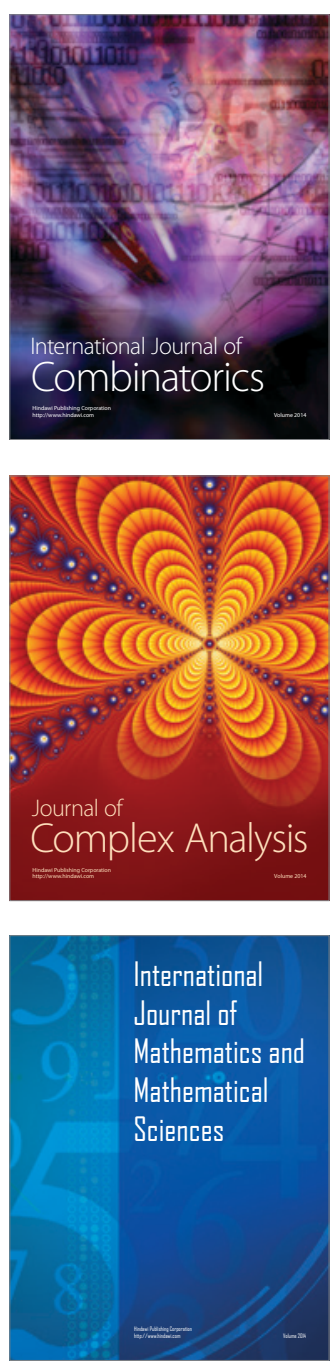
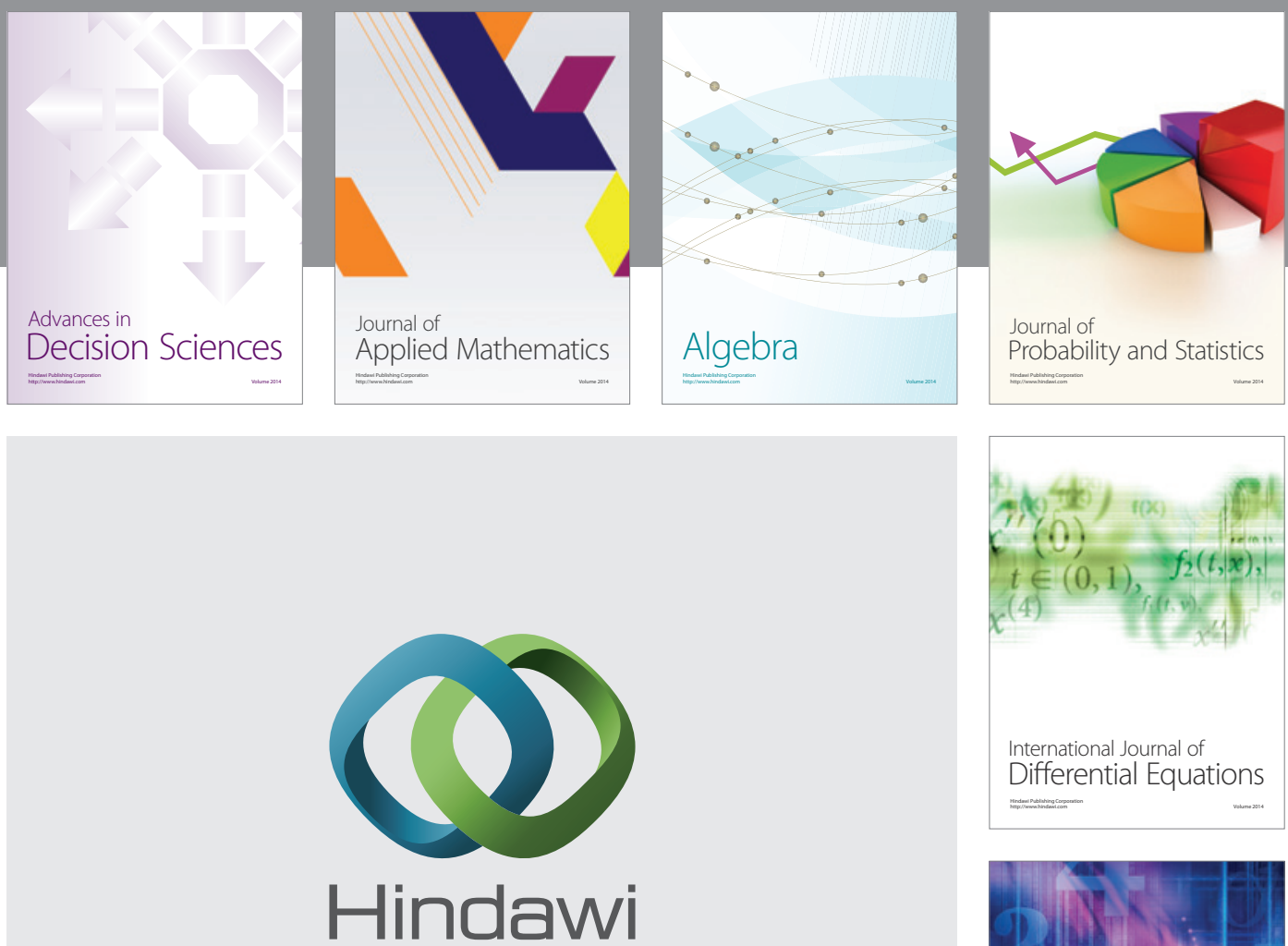

Submit your manuscripts at http://www.hindawi.com
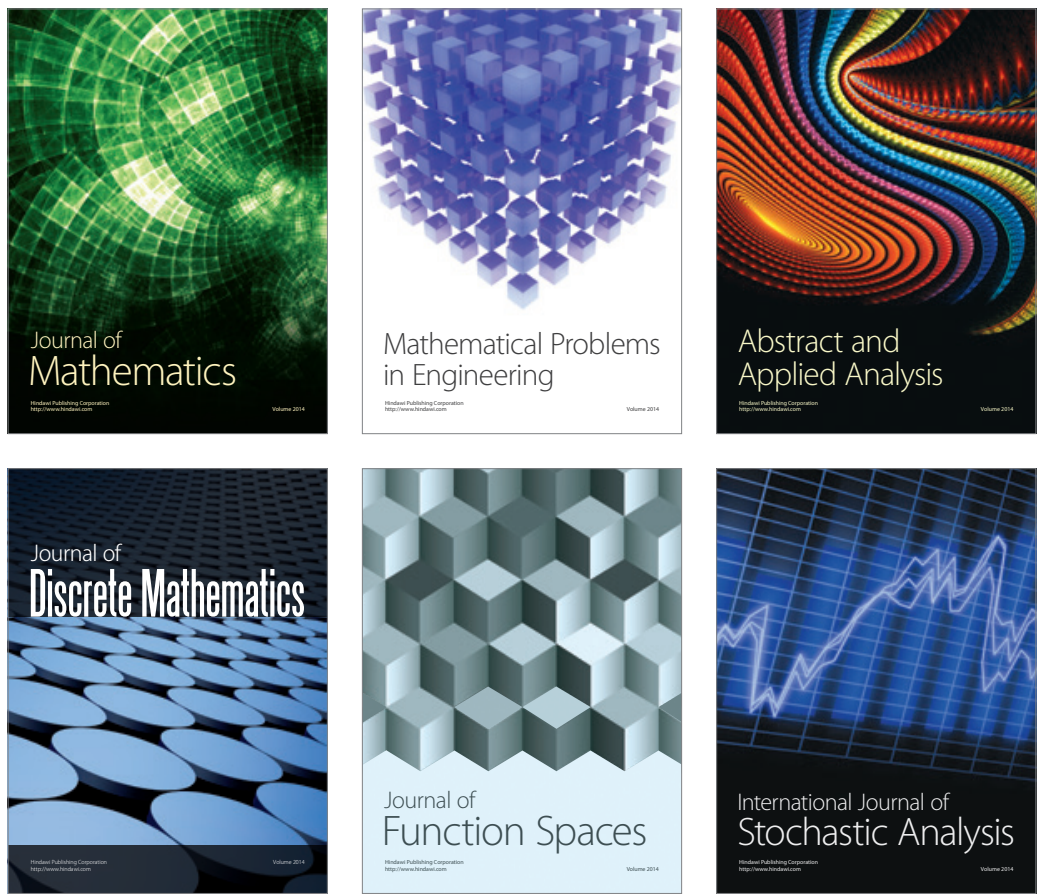

Journal of

Function Spaces

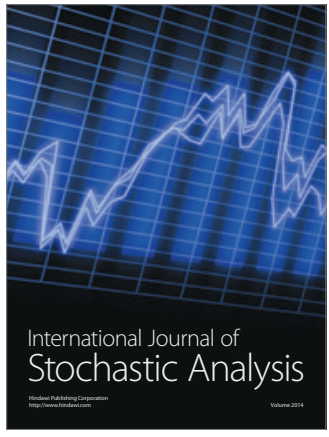

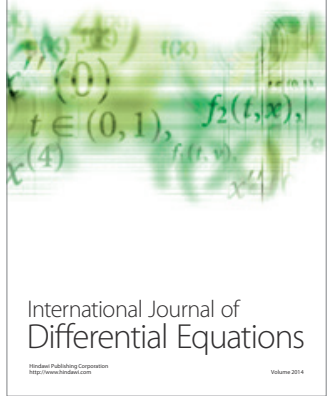
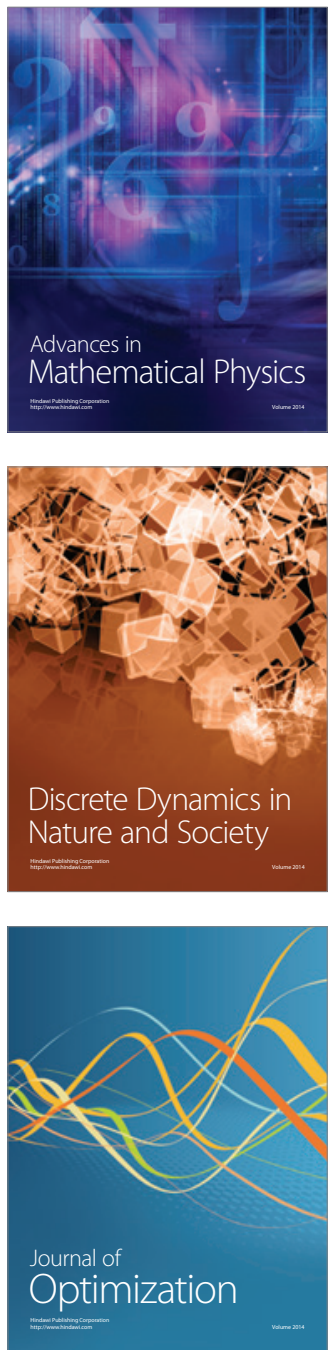